\title{
PSICODIAGNÓSTICO INTERVENTIVO COMO FERRAMENTA DE SUPORTE AO CONTROLE DA HETEROAGRESSIVIDADE
}

DOI: 10.22289/2446-922X.V7N2A20

\author{
Lorena Alves de Carvalho Lima ${ }^{1}$ \\ Larissa Gabriela Ferreira Brito \\ Dayse Chaves Lemos \\ Karoline Giele Martins de Aguiar
}

\section{RESUMO}

O presente artigo apresenta um estudo de caso clínico, a partir do relato de experiência, com objetivo de descrever a experiência de psicodiagnóstico interventivo com um indivíduo com sintomas de heteroagressividade e pensamentos intrusivos. A metodologia de estudo, deu-se, a partir da experiência do Estágio Supervisionado Básico em Psicologia e Processos de Avaliação e Diagnóstico, desenvolvidos em cinco sessões. Os procedimentos realizados foram: acolhimento, observação, escuta, entrevista psicológica (semiestruturada), testagem psicológica - Teste HTP (Casa - Árvore - Pessoa), para avaliação da personalidade, STAXI - 2 (Inventário De Expressão De Raiva Como Estado e Traço), para avaliação da expressão da raiva e entrevista devolutiva. Conclui-se, que o processo de psicodiagnóstico interventivo ocasionou melhora no quadro dos sintomas apresentados, a partir das intervenções, que facilitaram as expressões dos sentimentos e emoções. Permitiram a reflexão, autopercepção e tomada de consciência dos pensamentos invasivos e da heteroagressividade, possibilitando acesso às dificuldades vivenciadas, mobilizando recursos psíquicos para etapa de mudança.

Palavras-chave: Psicodiagnóstico; Avaliação Psicológica; Comportamento Agressivo.

\section{INTERVENTIVE PSYCHODIAGNOSIS AS A SUPPORT TOOL FOR HETEROAGRESSIVITY CONTROL}

\section{ABSTRACT}

This article is a clinical case study based on an experience report, with the aim of reporting the experience of interventional psychodiagnosis with an individual with symptoms of heteroaggression and intrusive thoughts. The case study methodology was based on the experience of the Basic Supervised Internship in Psychology and Assessment and Diagnosis Processes, the procedures performed were: reception, observation, listening, psychological interview (semi-structured), psychological testing - HTP Test (Home - tree - person), for personality assessment, STAXI - 2 (Anger Expression Inventory as State and Trait), for assessment of anger expression and feedback interview. It is concluded that the interventional psychodiagnostic process resulted in an

${ }^{1}$ Endereço eletrônico de contato: lorenaacarv@gmail.com

Recebido em 10/08/2021. Aprovado pelo conselho editorial para publicação em 21/10/2021.

Rev. Psicol Saúde e Debate. Out., 2021:7(2): 314-322. 
improvement in the symptoms, providing, from the operations, that facilitated the expression of feelings and emotions, allowing reflection, self-perception and awareness of invasive thoughts and heteroaggression, enabling access to difficulties experienced and thus mobilize psychic resources for the change stage.

Keywords: Psychodiagnosis; Psychological Assessment; Aggressive Behavior.

\section{PSICODIAGNÓSTICO INTERVENTIVO COMO HERRAMIENTA DE APOYO AL CONTROL DE HETEROAGRESIVIDAD}

\section{RESUMEN}

Este artículo es un estudio de caso clínico basado en un relato de experiencia, con el objetivo de relatar la experiencia del psicodiagnóstico intervencionista con un individuo con síntomas de heteroagresión y pensamientos intrusivos. La metodología del estudio de caso se basó en la experiencia del Internado Básico Supervisado en Psicología y Procesos de Evaluación y diagnóstico, los procedimientos realizados fueron: recepción, observación, escucha, entrevista psicológica (semiestructurada), testeo psicológico - Test HTP (Inicio - árbol - persona), para evaluación de la personalidad, STAXI - 2 (Inventário de expresión de ira como estado y rasgo), para evaluación de expresión de ira y entrevista de retroalimentación. Se concluye que el proceso de psicodiagnóstico intervencionista resultó en una mejoría de los síntomas, proporcionando, desde las operaciones, que facilitó la expresión de sentimientos y emociones, permitiendo la reflexión, autopercepción y conciencia de pensamientos invasivos y heteroagresión, posibilitando el acceso a las dificultades vividas. y así movilizar recursos psíquicos para la etapa de cambio.

Palabras Ilave: Psicodiagnóstico; Evaluación Psicológica; Comportamiento Agresivo.

\section{INTRODUÇÃO}

Atualmente, no Brasil existe um aumento significativo da produção científica, relacionada ao processo de Avaliação Psicológica (AP), após anos de crítica e questionamentos envolvendo a validade e precisão dos resultados, por falta de padronização e sistematização dos instrumentos utilizados. A partir da Resolução $n . .09 / 2018$, do Conselho Federal de Psicologia (CFP), obteve-se o resgate e avanços significativos na produção de testes psicológicos no país, com isso, progresso na AP, nas diversas áreas de atuação do profissional de psicologia (Reppold, \& Noronha, 2018).

A AP, é prática restrita ao profissional de psicologia, sendo a AP a expansão do desenvolvimento da prática do psicólogo, a partir da utilização de fontes de informações e de instrumentos que avaliam de forma consistente diversas demandas no indivíduo. A AP adentrou nos mais diversos contextos de atuação (Hutz, Bandeira, Trentini, \& Krug, 2016; Scaduto, Cardoso, \& Heck, 2019). Vale ressaltar, o movimento atual, que envolve a revogação da Resolução $n .{ }^{\circ}$ 02/2003, permitindo a comercialização de testes psicológicos a outras categorias de profissionais. O CFP, tem empenhado esforços de forma jurídica para resolutividade da questão e o não comprometimento do processo de AP (Alencar, Bezerra, \& Aguiar, 2021). 
Historicamente, dentre os contextos em que a AP tem mais divulgação e raízes epistemológicas, refere-se a clínica, sendo facilmente interpretada como psicodiagnóstico, ambas têm a mesma sistematização, porém tornam-se distintas pela finalidade (Hutz et al., 2016). O psicodiagnóstico é processo científico de investigação, assim como a AP. Ambos são realizados por profissionais com formação em psicologia, porém, a prevalência de ocorrência do psicodiagnóstico é no contexto clínico. Para a realização do processo é necessário a utilização de métodos e técnicas da ciência psicológica, objetivando o diagnóstico da demanda apresentada, e ao final do processo a entrevista devolutiva com a condução de intervenções futuras pertinentes ao caso (Hutz et al., 2016).

O psicodiagnóstico, é suscitado pela demanda individual, coletiva e/ou instituição, solicitado para as mais diversas finalidades. Sendo por muito tempo percebida como processo passivo por parte do profissional que investiga a demanda. No entanto, pode ser oportuno a prática de intervenções durante o processo. Sendo então, duas práticas realizadas pela psicóloga (o). A primeira, enquanto realiza o processo de avaliação, já descrito anteriormente, e a segunda é quando realiza intervenção focada na demanda inicial ou latente do paciente. Com isso, constata-se que em alguns atendimentos realizados no processo de psicodiagnóstico podem gerar mudanças, provocadas inicialmente pelo contato, gerando transformações (Ancona-Lopez et al., 1998; Huts et al., 2016; Milani, Tomael, \& Greinert, 2014).

Diante do avanço científico e histórico, o psicodiagnóstico passou a ser considerado um importante instrumento terapêutico, integrando a investigação e a possibilidade de entendimento dos mecanismos que produzem, possivelmente, adoecimento psíquico, a partir do uso de testes e técnicas psicológicas. Seu uso pode ser gerado por meio de uma proposta diagnóstica e interventiva que propicie mudanças, a partir da intervenção psicoterapêutica (Ancona-lopez et al., 1998; Andrade, \& Miranda, 2012).

Deve-se ressaltar, a necessidade do processo de intervenção, que seja pautado na consistência ética do profissional de psicologia, unindo a práxis a teórico-prática, que envolve a formação, considerando os demais aspectos que envolvem o processo. Por exemplo, considerando o ambiente de aplicação, condições do examinando, entre outros aspectos, necessários para a ocorrência e precisão do processo (Hutz, Bandeira, \& Trentini, 2015; Hutz et al., 2016). Ainda para Hutz et al. (2016), a AP concerne em uma complexidade de interações implicadas durante todo o processo, já que na análise, a partir da demanda, o indivíduo precisa ser compreendido em toda sua integralidade.

O psicólogo, ao ser requisitado para o processo de psicodiagnóstico, pode deparar-se com o indivíduo em sofrimento psíquico, fato que convoca o profissional a ter uma escuta empática, fato essencial para a compreensão da demanda. Essa compreensão possibilitará ao mesmo tempo, a apreensão do sofrimento e, ao mesmo tempo, fomentar a organização dos elementos narrados, que 
ocasionarão melhor na condução da própria dor (Braga, Mosqueira, \& Morato, 2012; Scaduto, Cardoso, \& Heck, 2019).

A partir do Estágio Supervisionado Básico em Psicologia e Processo de Avaliação Diagnóstica, realizado por estudantes de psicologia do $7 .$. período, o processo de desenvolvimento do psicodiagnóstico é uma das principais competências a serem desenvolvidas pelos discentes. $O$ processo de psicodiagnóstico, suscitou como possibilidade de conduta interventiva a luz de referencial teórico, como desfecho secundário ao processo, dar-se-á supressão de sintomas, como benfeitorias do psicodiagnóstico interventivo (Leila, 2007; Scaduto, Cardoso, \& Heck, 2019).

As demandas suscitadas podem ser diversas, desde um processo de autoconhecimento a sintomas de ansiedade. Estudos realizados em uma clínica escola, com crianças e adolescentes, apontam as principais demandas: dificuldades escolares, comportamento agressivo, dificuldade nas relações familiares e controle da impulsividade (Romaro, \& Capitão, 2003). Outro estudo em clínica escola, indica a predominância das demandas de sintomas depressivos, ansiedade, problemas familiares, agressividade, entre outros (Maravieski, \& Serralta, 2011).

A agressividade se apresenta como demanda recorrente nas clínicas escolas de Psicologia (Romaro, \& Capitão, 2003; Maravieski, \& Serralta, 2011). O comportamento agressivo, está associado a dificuldades de autocontrole e a pensamentos intrusivos desagradáveis (Gay, Schmidt, \& Van der Linden, 2011). De acordo com Costa (2016, apud Rachman, 2014), são pensamentos intrusivos, imagens ou impulsos indesejados que obstruem o fluxo natural da consciência e podem perturbar o comportamento em curso. Tais interferências ocorrem cotidianamente nos indivíduos e, podem ser facilmente descartadas. Porém, se o pensamento gera significância, sendo interpretada como algo inaceitável ou repugnante, este pode tornar-se perturbador e ter uma maior probabilidade de voltar, tornando assim as tentativas de controlar ou bloquear mais difíceis.

Pretende-se apresentar o processo psicodiagnóstico interventivo como ferramenta de instrumentação de estratégias terapêuticas para profissionais e acadêmicos de psicologia. Objetiva-se relatar a experiência de psicodiagnóstico a partir de um estudo caso com pensamentos intrusivos, atendido por estagiárias do 7. período de psicologia.

\section{MATERIAIS E MÉTODOS}

O caso clínico apresentado, constitui-se do relato de experiência do Estágio Supervisionado Básico em Psicologia e Processos de Avaliação e Diagnóstico, de alunos do $7^{\circ}$ período de Psicologia. O estágio ocorreu na Clínica Escola de uma Universidade, localizada no interior do Estado do Maranhão, entre os meses de setembro e novembro de 2020, com supervisão semanal. 


\section{PROCEDIMENTOS}

A paciente dirigiu-se a Clínica Escola Saúde e Bem-Estar de forma espontânea, voluntariando-se para a submissão no processo de Avaliação Psicológica, direcionada aleatoriamente para a estagiária autora do artigo. O método psicodiagnóstico interventivo proposto por Ancona-Lopez (1998), foi aplicado na pessoa avaliada. Todo o processo ocorreu em cinco sessões, com duração média de quarenta minutos, envolvendo os seguintes procedimentos: acolhimento, explanação e assinatura do Termo de Consentimento Livre e Esclarecido (TCLE), entrevista psicológica, testagem psicológica, observação e entrevista devolutiva.

\section{INSTRUMENTOS UTILIZADOS}

Teste HTP (Casa - Árvore - Pessoa), desenvolvido por Buck, em 1949, adaptado para versão brasileira e aprovação do Sistema de Avaliação de Testes Psicológicos - SATEPSI em 2003. Um teste projetivo que avalia a personalidade humana a partir de três desenhos (Casa - Árvore Pessoa) na sequência realizado inquérito sobre os desenhos, não tendo limite de tempo para a aplicação.

Teste STAXI - 2 (Inventário de Expressão de Raiva como Estado e Traço), desenvolvido por Spielberger em 2010, validado e aprovado pelo SATEPSI em 2011. O instrumento contém 57 itens, escala tipo Likert, para avaliação de traços de expressão da raiva. Não possui limite de tempo, com média de tempo de aplicação, de 12 há 15 minutos.

\section{PROCEDIMENTOS ÉTICOS}

O estudo ocorreu conforme as diretrizes e os preceitos da Resolução $n . .9510 / 2016$, do Conselho Nacional de Saúde (CNS, 2016), aprovado pelo Comitê de Ética e Pesquisa (CEP), sob o número n.. 4.742.382. Desse modo, a paciente decidiu participar do processo de avaliação psicológica, assinando o Termo de Consentimento Livre e Esclarecido (TCLE), que descrevia os procedimentos de sigilo da pesquisa, guarda do material e possíveis estudos posteriores.

\section{RELATO DA EXPERIÊNCIA}

Nas primeiras sessões a paciente demonstrou adesão ao relatar sobre o histórico familiar, curso dos episódios das crises de ansiedade associadas a pensamentos de agressividade. $O$ entrevistador, a partir do momento que acolhe a demanda verbalizada, permite a aproximação entre o mesmo e a dor do outro, que solicita ajuda. A demanda inicial verbalizada, mesmo que não seja 
a demanda latente, ocasiona a validade do sofrimento, permitindo maior adesão a proposta interventiva e, ao mesmo tempo, o indivíduo vai apropriando-se, podendo ainda organizar melhor suas demandas (Cerioni, \& Herzberg, 2016).

No tratamento de pessoas com Transtornos Mentais a adesão torna-se relevante, diante dos cuidados disponibilizados na Instituição de Tratamento de referência, e na continuidade do autocuidado em seu lar e nas relações laborais, interpessoais e comunitárias. De acordo com o Manual diagnóstico e estatístico de transtornos mentais (DSM-V, 2014) a adesão ao tratamento é algo essencial para a condução do tratamento.

Logo nas duas primeiras sessões evidenciou-se, medo e angústia a partir da autopercepção sobre seus sentimentos, pensamentos e comportamentos agressivos direcionados a membros de sua família. O comportamento agressivo, pode ser entendido como ação do indivíduo direcionada, com intencionalidade de acarretar algum prejuízo, seja físico ou psicológico a outro indivíduo, ou grupo. Todavia, não pode ser considerado e classificado como patológico quando o indivíduo consegue manter o controle das ações, mediante os contextos que provocam tais comportamentos (Dodge, \& Coie, 1987; Landim, \& Borsa, 2017).

A partir da demanda apresentada, associada a observação e a análise inicial da estagiária, optou-se pela bateria de testes psicológicos, que contemplassem a complexidade do caso. Os instrumentos utilizados foram: HTP (Casa - Árvore - Pessoa), para avaliação da personalidade e STAXI - 2 (Inventário de Expressão de Raiva como Estado e Traço), para avaliação da expressão da raiva, ambos com parecer favorável pelo Sistema de Avaliação dos Testes Psicológicos (Satepsi).

No teste de personalidade HTP, durante o inquérito, a paciente relatou de forma intensa a infância, relembrando e relatando a forma como viveu e como gostaria de tê-la vivenciado. Em decorrência da maternidade precoce, os relatos da infância podem estar associados ao fato de a mencionada não ter se desenvolvido, a partir dos ciclos vitais, de maneira completa, no qual assumiu responsabilidades adultas ainda jovem. Em um estudo realizado com 201 pessoas que buscaram atendimento psicoterápico em uma instituição de formação, revelou que $88 \%$ da amostra sofreu abuso emocional e/ou negligência emocional na infância (Waikamp, \& Barcellos 2018). Os dados sugerem a internalização da experiência infantil, associados a sintomas psicopatológicos na vida adulta, somados a maternidade precoce.

O desenvolvimento infantil quando permeado por situações de risco, podem refletir no desenvolvimento de agravos nas fases posteriores do clico vital, como na adolescência ou fase adulta (Maia, \& Albuquerque, 2005). A apresentação de comportamentos agressivos desde a infância, quanto mais precoces as ocorrências, maior a probabilidade de resultarem em problemas de agressividade e outros comportamentos hostis ao longo da vida do indivíduo (Landim, \& Borsa, 2017). Já os comportamentos agressivos desempenham forte influência em relação ao 
desenvolvimento pessoal e nas relações em grupos. A fase inicial da vida de uma criança é de suma importância para o desenvolvimento da sua personalidade, adequação social e psicológica (Joly, Dias, \& Marini, 2009).

Aspectos analisados a serem destacados foram; sentimento de insegurança, retraimento, descontentamento, indicando possível quadro de depressão, falta de apoio e fixação no passado (Buck, 2009). Podem ser indicativos de episódios depressivos leves, moderados, ou graves típicos, pois os pacientes acometidos sofrem de rebaixamento do humor, diminuição da energia e a atividade é diminuída. Em relação às capacidades emocionais como: sentir prazer, interesse e concentração, passam por um processo de diminuição e/ou rebaixamento (Fonseca, Coutinho, \& Azevedo, 2008).

Os sentimentos relacionados a raiva, a partir do STAXI-2, indicam que os resultados gerais obtidos pela paciente, encontraram-se dentro média normal da amostra. Entretanto, quatro traços estavam na zona elevada. Foram esses: Vontade de Expressar Raiva Verbalmente (E-RV), Vontade de Expressar Raiva Fisicamente (E-RF), Traço de raiva (TrR) e Índice de Expressão de Raiva (IEB). Tais traços, podem estar relacionados aos episódios de ansiedade, associados a pensamentos de raiva e ideação de comportamentos agressivos direcionados ao filho. Em relação ao Estado de Raiva, os resultados revelam que a mencionada está localizada no limite da zona média. Isso demostra que no momento da avaliação a paciente não estava vivenciando sentimentos de raiva, mas possivelmente ao ficar exposta ao contato direto com o filho poderia experienciar tais sentimentos.

\section{CONSIDERAÇÕES FINAIS}

A partir do caso clínico exposto foi possível identificar que os traços de raiva podem estar relacionados aos pensamentos intrusivos, que de acordo com Julien, O'Connor e Aardema (2007, apud Yao et al., 1999) se caracterizam por pensamentos, imagens ou impulsos repetitivos, desagradáveis e involuntários que podem aparecer brutalmente em consciência. As intervenções ocorridas durante o psicodiagnóstico permitiram que a paciente pudesse tomar consciência de suas dificuldades e assim mobilizar recursos psíquicos de mudança.

Em relação aos pensamentos intrusivos estarem direcionados ao filho, observou-se possível relação com a maternidade precoce. Os relatos da infância poderiam estar associados ao fato de a paciente não ter vivenciado os ciclos de sua vida de maneira completa, pois, passou a ter responsabilidades muito cedo.

Dessa forma, foi possível observar dificuldades na convivência psicossocial, característico de pessoas introvertidas, bem como também traços de expressão de raiva. Foi recomendado que 
a paciente desse continuidade nas sessões de psicoterapia e psiquiatria, para fins de autoconhecimento e desenvolvimento de habilidades sociais de autocontrole.

\section{REFERÊNCIAS}

Alencar, P. H. S., do Carmo Bezerra, N., de Aguiar, K. G. M. (2021). Relato de Experiência: Processo de Avaliação Psicológica em um Centro De Referência de Assistência Social (CRAS). Psicologia e Saúde em debate, 7(1), 268-279. DOI: 10.22289/2446-922X.V7N1A19

American Psychiatric Association. (2014). DSM-5: Manual diagnóstico e estatístico de transtornos mentais. Artmed Editora.

Ancona-lopez, M. (org.) (1998). Psicodiagnóstico: processo de intervenção. São Paulo: Cortez.

Braga, T. B. M., Mosqueira, S. M., Morato, H. T. P. (2012). Cartografia clínica em plantão psicológico: investigação interventiva num projeto de atenção psicológica em distrito policial. Temas em Psicologia, 20(2), 555-569. Recuperado em 09 de março de 2021 de: doi: 10.9788/TP2012.2-20.

Buck, J. N. H-T-P: Casa - Árvore - Pessoa, (2009). Técnica Projetiva de Desenho: Manual e Guia de Interpretação. (2 $2^{\underline{a}}$ ed.). São Paulo: Vetor.

Cerioni, R. A. N., Herzberg, E. (2016). Expectativas de pacientes acerca do Atendimento Psicológico em um Serviço-Escola: da Escuta à Adesão. Psicologia: Ciência e Profissão, 36, 597-609. DOI: 10.1590/1982-3703001402014.

Costa, D. A. J. (2016). Pensamentos intrusivos desagradáveis: frequência, avaliação e estratégias para os controlar (Doctoral dissertation). Recuperado em 09 de março de 2021 de:https://sapientia.ualg.pt/bitstream/10400.1/9962/1/Daniela-2016-PensamentosIntrusivos.pdf.

Andrade, A. M., Miranda, C. E. S. (2012). Psicodiagnóstico interventivo como modalidade de atuação terapêutica: reflexões a partir de um caso clínico. Revista Kaleid oscópio, 3, 59-76. REC:

Dodge, K. A., Coie, J. D. (1987). Social-information-processing factors in reactive and proactive aggression in children's peer groups. Journal of personality and social psychology, 53(6), 1146.

Fonseca, A. A. D., Coutinho, M. D. P. D. L., Azevedo, R. L. W. D. (2008). Representações sociais da depressão em jovens universitários com e sem sintomas para desenvolver a depressão. Psicologia: Reflexão e Crítica, 21, 492-498. Recuperado em 09 de março de 2021 de: https://doi.org/10.1590/S0102-79722008000300018.

Gay, P., Schmidt, R. E., Van der Linden, M. (2011). Impulsivity and intrusive thoughts: Related manifestations of self-control difficulties?. Cognitive Therapy and Research, 35(4), 293-303. DOI: https://doi.org/10.1007/s10608-010-9317-z.

Hutz, C. S., Bandeira, D. R., Trentini, C. M. (2015). Psicometria. Artmed Editora.

Hutz, C. S., Bandeira, D. R., Trentini, C. M., Krug, J. S. (2016). Psicodiagnóstico. Porto Alegre: Artmed, 27. 
Joly, M. C. R. A., Dias, A. S., Marini, J. A. D. S. (2009). Avaliação da agressividade na família e escola de ensino fundamental. Psico-USF, 14(1), 83-93.

Julien, D., O'Connor, K. P., Aardema, F. (2007). Intrusive thoughts, obsessions, and appraisals in obsessive-compulsive disorder: A critical review. Clinical Psychology Review, 27(3), 366-383. Recuperado em 09 de março de 2021 de: doi:10.1016/j.cpr.2006.12.004 Rev. Psicol Saúde e Debate. Jan., 2021:7(1): 1-14.

Landim, I., Borsa, J. C. (2017). Revisão sistemática sobre programas de intervenção para redução de comportamentos agressivos infantis. Contextos clínicos, 10(1), 110-129. Recuperado em 09 de março de 2021 de:doi: https://doi.org/10.4013/ctc.2017.101.09

Leila, S. (2007). Psicodiagnóstico interventivo: uma proposta de ensino em Atendimento Clínico. Mudanças-Psicologia da Saúde, 15(2), 128-134.

Maia, J. M. D., de Albuquerque Williams, L. C. (2005). Fatores de risco e fatores de proteção ao desenvolvimento infantil: uma revisão da área. Temas em psicologia, 13(2), 91-103. Recuperado em 09 de março de 2021 de:https://www.redalyc.org/pdf/5137/513751425002.pdf.

Maravieski, S., Serralta, F. B. (2011). Características clínicas e sociodemográficas da clientela atendida em uma clínica-escola de psicologia. Temas em Psicologia, 19(2), 481-490. Recuperado de: https://www.redalyc.org/pdf/5137/513751438011.pdf.

Milani, R. G., Tomael, M. M., Greinert, B. R. M. (2014). Psicodiagnóstico interventivo psicanalítico. Estudos interdisciplinares em Psicologia, 5(1), 80-95. DOI: 10.5433/2236-6407.2014v5n1p80.

Reppold, C. T., Noronha, A. P. P. (2018). Impacto dos 15 anos do Satepsi na avaliação psicológica brasileira. Psicologia: Ciência e Profissão, 38(SPE), 6-15. DOI: https://doi.org/10.1590/19823703000208638.

Romaro, R. A., Capitão, C. G. (2003). Caracterização da clientela da clínica-escola de psicologia da Universidade São Francisco. Psicologia: teoria e prática, 5(1), 111-121. Recuperado de: http://pepsic.bvsalud.org/pdf/ptp/v5n1/v5n1a09.pdf.

Scaduto, A. A., Cardoso, L. M., Heck, V. S. (2019). Modelos interventivo-terapêuticos em avaliação psicológica: estado da arte no Brasil. Avaliaçao Psicologica: Interamerican Journal of Psychological Assessment, 18(1), 67-75.

Spielberger. C. D, (2010). Staxi-2: Inventário de Raiva como Estado e Traço. (1ำed.) São Paulo: Vetor.

Waikamp, V., Barcellos Serralta, F. (2018). Repercusiones del trauma en la infancia en la psicopatología de la vida adulta. Ciências Psicológicas, 12(1), 137-144. Recuperado em 09 de março de 2021 de: doi: 10.22235/cp.v12i1.1603. 